\title{
Sleep Quality, Quality of Life and Insomnia Among Patients with Chronic Low Back Pain Compared to Normal Individuals
}

\author{
Mahnaz Abbasi ${ }^{1}$, Amir Mohammad Kazemifar ${ }^{2}$, Hasti Fatorechi ${ }^{3}$, Zohreh Yazdi ${ }^{4}$ \\ ${ }^{1}$ Associate professor of Rheumatology, Metabolic Diseases Research center, Qazvin University of medical sciences, Qazvin, Iran \\ ${ }^{2}$ Associate professor of Qazvin University of medical sciences, Qazvin, Iran \\ ${ }^{3}$ General Physician, Qazvin University of medical sciences, Qazvin, Iran \\ ${ }^{4}$ Associate professor of Occupational medicine, Social Determinant of Health, Qazvin University of medical sciences, Qazvin, Iran
}

\begin{abstract}
Objective: Low back pain may affect various aspects of quality of life including sleep.

There are some reports about prevalence of sleep disorders in patients with chronic low back pain. The present study was conducted to estimate sleep quality, quality of life, and insomnia in patients with chronic low back pain compared to age and sex matched control group.

Methods: The present case-control study was performed on 140 persons (70 patients with chronic low back pain and 70 persons as control group). They were asked to fill Pittsburg questionnaire for evaluation of sleep quality, SF-36 questionnaire for evaluation of quality of life, and insomnia questionnaire. Also, the severity of their back pain was assessed using visual analogue scale (VAS).

Results: Mean age of the studied persons was $49.88 \pm 11$ and $48.33 \pm 10$ years in the case and control groups, respectively ( $P$-value $>0.05)$. Thirty one persons of each group were male. Individuals in the case group had worse sleep quality (correlation coefficient 0.478 with severity of pain), more severe insomnia (correlation coefficient 0.386 with severity of pain), and lower quality of life (correlation coefficient 0.665 with severity of pain) compared to the control group.

Conclusion: Results of the present study showed that patients with chronic low back pain have higher prevalence of insomnia, lower quality of life, and worse sleep quality when judged against control group. Also, this case-control study clearly showed the magnitude of the relationship between them.
\end{abstract}

Keywords: sleep quality; quality of life; insomnia; low back pain

\section{INTRODUCTION}

Low back pain is a common complaint of many patients who seek medical care. Sixty to eighty percent of human being experience low back pain at least one time in their life (Freburger et al., 2009). Low back pain is the most

*Correspondence: dr.zyazdiayahoo.com

Zohreh Yazdi, Associate professor of Occupational medicine, Social

Determinant of Health, Qazvin University of medical sciences, Qazvin, Iran

Received: 05 August 2017 Accepted: 23 October 2017

Sleep and Hypnosis

Journal homepage:

www.sleepandhypnosis.org

ISSN:1302-1192 (Print) 2458-9101 (Online) common chronic pain, too. If low back pain persists for more than 12 weeks, it is called chronic low back pain (Agmon, \& Armon 2014; Kornu, Dao, Orozco, \& Patel 2011).

Insomnia or inadequate sleep is also a common problem in developed countries. It is presented as difficulty in start of the sleep, frequent awakening during night with difficulty in return to sleep, and too early awakening (Alsaadi et al., 2013). Sixty four million of Americans suffer from inadequate sleep according to report of the health ministry of USA. This is 1.4 time more prevalent in women (Basics 2011; Lamberg 2007). It is suggested that persons with sleep problems may have higher sensitivity to pain. Chronic pain may become 
complicated in them (Alsaadi, McAuley, Hush, \& Maher 2011; Bahouq, Allali, Rkain, Hmamouchi, Hajjaj-Hassouni 2013). Pain and sleep may have intricate relationship with each other. So, association between pain and sleep quality is expected (Hurley et al., 2008). On the other hand, sleep quality has closed relationship with quality of life. Poor sleep quality adversely affect mood, pain experience, daily performance, and general quality of life. There are many reports of relationship between pain and mood in patients with low back pain. Sleep deprivation and poor sleep quality diminish pain threshold and emotional capacity to deal with pain by the patient (DiplPsych, Jürgen-Christian, \& Wolfgang 2004; van de Water, Eadie, \& Hurley 2011). Nevertheless, the exact causation of relationship between sleep and back pain is not clear. Poor sleep may predispose the person to low back pain. But, low back pain may also produce sleep disturbance (Agmon , \& Armon 2014; Alsaadi 2011; Marty et al., 2008). Cognitive behavioral therapy may alleviate low back pain and other chronic pains (Smith, \& Haythornthwaite 2004). Also, rehabilitative therapy may help the patients with low back pain according to cohort and case-control studies (van de Water et al., 2011).

The present study was aimed to evaluate sleep quality, quality of life, and insomnia in patients with low back pain compared to normal individuals.

\section{METHODS}

The present case- control study was performed in the rheumatology OPD clinic of a teaching referral hospital, Qazvin, Iran in 2014-2015. The attended patients with chronic low back pain were included in the study as the case group. The patients with previous history of any systemic disease, neurologic diseases, trauma to vertebral column, pregnant patients, and patients with acute low back pain (less than 4 weeks duration) or sub- acute pain (4-12 weeks duration) were excluded from the study. The control group was selected from the attendants of the same clinic with no history of low back pain during last 3 months. Their age and gender were matched to the case group.

The patients in both case and control group were examined by one of the authors. Their age, gender, body weight, past medical history, and drug history were recorded. They were asked to fill Pittsburg questionnaire for evaluation of sleep quality, SF-36 questionnaire for evaluation of quality of life, and insomnia questionnaire. Also, the severity of their back pain was assessed using visual analogue scale (VAS) to assess their perception of pain severity from 0 to 100 (Boonstra, Preuper, Balk, \& Stewart 2014; Crichton 2001).

The SF-36 questionnaire has eight distinct scores which the first 4 measures the physical aspects of quality of life and the other 4 scores measures its emotional scores. The questionnaire has been previously used for evaluation of quality of life in persons with low back pain in earlier studies (O'Donoghue, Fox, Heneghan, \& Hurley 2009; Masumian, Shairi, \& Hashemi 2013).

The Pittsburg questionnaire is a comprehensive tool for evaluation of the recent sleep quality. It comprises 19 items which are measured with 4 scores Likert criteria. It has 7 sub- heading which assess subjective quality of sleep, lag in starting the sleep, sleep duration, efficiency of the sleep, sleep disorders, use of hypnotic drugs, and disorders of daily functions. The final score ranged from 0 to 21. The higher score of the questionnaire means poorer sleep quality. Any score higher than 5 is regarded as poor sleep quality. The validity of the questionnaire has been confirmed in the earlier studies (Alsaadi et al., 2013; Nasab, \& Azimi 2008).

The insomnia severity questionnaire has 5 questions which estimate the severity of insomnia during last 2 weeks. Its score ranges from 0 to 28 . The higher score demonstrates the higher risk of insomnia and need for medical assistance. The questionnaire was used by Morine and his coworkers for the first time (Alsaadi et al., 2013). The Kronbach' alpha of the questionnaire was 0.76 in the current study which is acceptable.

The collected data was entered to SPSS software version 19. The mean and standard deviation were determined for the variables. The independent T-test was used to compare the variables in the case and control groups on account of normal distribution of the data. The Chi- square test was used to compare the qualitative variables. Any P-value less than 0.05 were regarded statistically significant. 


\section{RESULTS}

Seventeen patients were evaluated in the present study including 39 (55.7\%) female and 31 (44.3\%) male. There was no statistical difference in sex ratio and age and weight distribution between the groups as it has been demonstrated in table 1 . The groups differed in their height distribution. BMI was higher in the case group compared to the control group. The groups had no difference in cigarette smoking habit (8.6\% in patient group and $15.7 \%$ in control group. P-value $=0.196$ ).

The score of physical aspect of quality of life was $40 \pm 14.6$ and $38.6 \pm 9.9$ in the case and control groups respectively. The difference is statistically significant (table 2). The patients in the case group had low back pain for averagely 4.8 years (ranged from 5 months to 14 years). Eighty five percent of them have been using sedative drugs to control their pain. Their mean VAS score was 60.94 which means their pain severity is higher than moderate.

The case group had higher scores in Pittsburg and insomnia severity questionnaire compared to the control group (table 2). Fifty patients (71\%) in the case group and only 5 persons in thecontrol group had poor sleep quality. Thirty patients (43\%) in the case group had moderate insomnia (score 15-21), while only 6 persons in the control group had the same sore (table 2).

The index of sleep quality was significantly higher in the case group $(11.2 \pm 4.5$ vs. $3.7 \pm 1.6)$. The sore was markedly high in some patients in the case group, particularly in some sub- scores (table 2). For example, the proportion of patients with awakening during night at least 3-4 times in a week was $77.1 \%$ in the case group and $24.3 \%$ in the control group (P-value less than 0.001 ). Also, the ratio of inadequate sleep for 3-4 weeks due to need for voiding were $51 \%$ and $20 \%$ in the case and control groups respectively (P-value less than 0.001). Moreover, the ratio of inability to start sleep for 30 minutes was $44.44 \%$ in the case group and $18.6 \%$ in the control group (P-value less than 0.001$)$. Twenty five patients (35.7\%) in the case group had difficulty to maintain their arousal for daily social activity at least 3-4 times in 1 week (table 3). The corresponding ratio was $5.7 \%$ in the control group (P-value less than 0.001). Moreover, $34.3 \%$ in the case group (vs. $8.6 \%$ in the control group) needed hypnotic drugs to go to sleep (P-value less than 0.001 ). Also, $65 \%$ in the case group and $38 \%$ in the control group had work problem (table 3).

Table 1. Demographic characteristics in low back pain patients and control groups

\begin{tabular}{|c|c|c|c|}
\hline Variables & Patient group & Control group & p-value \\
\hline Gender (male/female) \% & $39 / 31(55.7 \%)$ & $39 / 31(55.7 \%)$ & - \\
\hline Age (mean $\pm S D)$ & $49.88(11.61)$ & $48.34(10.23)$ & 0.406 \\
\hline Weight (mean \pm SD) & $72.18(9.64)$ & $68.28(14.81)$ & 0.067 \\
\hline Height (mean \pm SD) & $161.3(8.1)$ & $168.7(8.6)$ & 0.004 \\
\hline $\mathrm{BMI}(\mathrm{kg} / \mathrm{m} 2)$ & $27.83(1.2)$ & $24.77(2.4)$ & 0.001 \\
\hline Physical activity more than 0.5 hour per day & $95.7 \%(67)$ & $85.7 \%(60)$ & 0.042 \\
\hline Total sleep time (mean \pm SD) & $5.85(0.96)$ & $6.64(1.96)$ & 0.003 \\
\hline Number of awakening per night & $3.22(0.7)$ & $1.29(0.3)$ & $<0.001$ \\
\hline Time of awakening (min) & $71.2(9.4)$ & $35.25(4.5)$ & $<0.001$ \\
\hline Sleep efficiency & $0.83(0.1)$ & $0.92(0.2)$ & $<0.001$ \\
\hline
\end{tabular}

Table 2. Subjects' answer about the questionnaires

\begin{tabular}{|c|c|c|c|}
\hline & Patient group & Control group & p-value \\
\hline Mental health subscale of SF-36 (mean \pm SD) & $38.6 \pm 9.9$ & $58.6(4.3)$ & $<0.001$ \\
\hline Physical functioning subscale of SF-36 (mean \pm SD) & $40(14.6)$ & $56.2(4)$ & $<0.001$ \\
\hline Insomnia severity index (0-28) & $14.5(8.3)$ & $2.5(2.2)$ & $<0.001$ \\
\hline PSQI (0-21) & $11.2(4.5)$ & $3.7(1.6)$ & $<0.001$ \\
\hline Visual analogue scale (mean $\pm S D)$ & $60.94(30.51)$ & - & - \\
\hline
\end{tabular}


Table 3. Differences in sleep parameters between patients and control groups

\begin{tabular}{|c|c|c|c|c|}
\hline & Patient group & Control group & $p$-value & $\begin{array}{l}\text { Pearson's } \\
\text { chi-square }\end{array}$ \\
\hline Awakening during the night & $54(77.1 \%)$ & $17(24.3 \%)$ & $<0.001$ & 39.76 \\
\hline Get up to use the bathroom & $36(51.4 \%)$ & $14(20 \%)$ & 0.001 & 15.61 \\
\hline Cannot get to sleep within 30 minutes & $31(44.3 \%)$ & $13(18.6 \%)$ & 0.004 & 13.45 \\
\hline Having pain during night & $38(54.3 \%)$ & $4(4.3 \%)$ & $<0.001$ & 48.40 \\
\hline Difficulty to stay awake for social activities & $25(37.5 \%)$ & $5(5.7 \%)$ & $<0.001$ & 34.58 \\
\hline Using sleep medication & $24(34.3 \%)$ & $6(8.6 \%)$ & 0.001 & 15.46 \\
\hline Lack of enough motivation at work & $46(65)$ & $27(38 \%)$ & 0.013 & 10.77 \\
\hline
\end{tabular}

*Data are presented as number and percentage.

Table 4. Differences in PSQI parameters between two groups

\begin{tabular}{lccc}
\hline & Patient group & Control group & p-value \\
\hline Subjective sleep quality & $0.7 \pm 1.3$ & $0.5 \pm 0.8$ & 0.04 \\
Delay in initiation of sleep & $0.8 \pm 0.4$ & $0.4 \pm 1.1$ & 0.03 \\
Sleep duration & $0.8 \pm 0.3$ & $0.5 \pm 0.4$ & 0.04 \\
Sleep satisfaction & $1.1 \pm 0.5$ & $0.7 \pm 0.6$ & 0.02 \\
Sleep disturbance & $0.9 \pm 0.7$ & $0.6 \pm 0.3$ & 0.04 \\
Use of hypnotic drugs & $0.7 \pm 0.5$ & $0.5 \pm 0.4$ & 0.04 \\
Disturbance in daytime activities & $0.9 \pm 1.2$ & $0.5 \pm 0.5$ & 0.01 \\
\hline
\end{tabular}

The pain score (VAS score) had statistically positive correlation with sleep quality score ( $P$-value less than $0.001 ; r=0.478)$, insomnia score (P-value less than 0.001 ; $r=0.386$ ), and quality of life (P-value less than $0.001 ; r=$ 0.665 ) according to Pearson correlation coefficient. In other words, as the pain intensifies, the quality of sleep, insomnia, and quality of life worsen.

As it has been demonstrated in table 4, the patients in the case group had higher scores in all sub-headings of the Pittsburg questionnaire which confirms their poorer sleep quality.

\section{DISCUSSION}

The present study was conducted to evaluate sleep quality, insomnia, and quality of life in patients with chronic low back pain. We showed that these patients have higher prevalence of insomnia, poorer quality of life, and inferior sleep quality compared to normal individuals. We also confirmed that the pain severity has moderate correlation with sleep quality and quality of life, and has weak correlation with insomnia. Similarly, Alsaadi has suggested that the pain severity in low back pain correlate weakly with insomnia (Alsaadi et al., 2011). There are various factors that are connected with insomnia. Pain with any origin is only one of them. Similarly, Bahoug and his coworkers has found also moderate correlation between the pain severity and insomnia (Bahouq et al., 2013).

We found that persons with low back pain had statistically significant lower mean sleep duration and more sleep awakening during night. These findings are similar to the study of van de Water and his colleagues (van de Water, Eadie, \& Hurley 2011). Patients with low back pain had more prolonged delay before start of sleep, lower sleep duration, and lower sleep efficiency (lower than $85 \%$ which is considered optimal). It is opposed to the study of Buysse that has reported more than $85 \%$ efficiency of sleep in patients with low back pain (Buysse et al., 2006). But, it is in agree with study of O ' Donoghue (O'Donoghue, Fox, Heneghan, \& Hurley 2009).

There was no difference between the studied groups in the frequency of cigarette smoking in the current study. However, it is claimed that patients with low back pain had higher prevalence of cigarette smoking when compared with normal population (Orhurhu, Pittelkow, \& Hooten 2015). The exact basis for this finding is unclear, if 
it is considered correct. Different biological mechanisms from neurological to biomechanical have been hypothesized for this relationship. For example, nicotine cigarette causes change in the perception and threshold for pain that increasing the self-reporting of pain (Green et al., 2016).

Patients with low back pain had more severe insomnia in the present study. Consequently, they had higher use of hypnotic drugs, poorer arousal for their daily activity and more work conflicts. So, they need clinical support and consult to deal with the problem. It is concordant with the studies of Alexander in Ireland, Marti in France, and $O$ ' Donoghue (van de Water et al., 2011; Marty et al., 2008; O'Donoghue, Fox, Heneghan, \& Hurley 2009).

Patients with low back pain had lower score in quality of life in the present study. It agrees with the study of Bentson (Orhurhu, Pittelkow, \& Hooten 2015). Both physical and emotional scores were affected. Therefore, not only physical consequences of low back pain, but also its emotional effects are involved to result in low quality of life in such patients (Orhurhu, Pittelkow, \& Hooten 2015; Bentsen et al., 2008).

Relationship between low back pain and sleep problems is not clarified yet. The results of the related studies are not consistent. In some studies, sleep problems has been considered as risk factor for development of low back pain (Agmon, \& Armon 2014). Yet, some others believe that any pain producing condition affects on sleep problems, insomnia, and quality of life in an unspecific manner (Afkham Ebrahimi et al., 2008). It can be hypothesized that sleep deprivation may lower the pain threshold in normal individuals. So, sleep deficiency declines the emotional capacity to deal with the chronic

\section{References}

Afkham Ebrahimi, A., Bandi, G., Salehi, M., Tafti, K., Vakili, Y., Farsi, A. (2008) Sleep parameters and the factors affecting the quality of sleep in patients attending selected clinics of Rasoul-eAkram Hospital. Razi Journal of Medical Sciences, 15(58), 31-8.

Agmon, M., Armon, G. (2014) Increased Insomnia Symptoms Predict the Onset of Back Pain among Employed Adults. PloS One, 9(8), e103591.

Alsaadi, S. M., McAuley, J. H., Hush, J. M., Maher, C. G. (2011) Prevalence of sleep disturbance in patients with low back pain. European Spine Journal, 20(5), 737-43. pains which in turn influences the quality of life (Marty et al., 2008). Physicians who treat patients with low back pain should be notice this interrelationship and refer their patients for management of their possible sleep problems.

\section{CONCLUSION}

The patients with low back pain may suffer from insomnia, poor sleep quality and poor quality of life. Also, this case-control study clearly showed the magnitude of the relationship between them. These problems should be taken into account in management of such patients. Report of insufficient sleep may be predictive of chronic back pain which in turn may affect the patient's quality of sleep. The physicians who treat such patients should notice probability of sleep disorders in these patients and should refer them to the related specialist, if needed. The proper management of these problems may help the patient to deal suitably with their original intolerable trouble.

\section{Funding: None}

Conflict of interest: Authors declare there is no conflict of interest.

Ethical approval: All procedures performed in studies involving human participants were in accordance with the ethical standards of the institutional and/or national research committee and with the 1964 Helsinki declaration and its later amendments or comparable ethical standards.

Alsaadi, S. M., McAuley, J. H., Hush, J. M., Bartlett, D. J., Henschke, N., Grunstein, R. R., et al. (2013) . Detecting insomnia in patients with low back pain: accuracy of four self-report sleep measures. BMC Musculoskeletal Disorders, 14(1), 196.

Bahouq, H., Allali, F., Rkain, H., Hmamouchi, I., Hajjaj-Hassouni, N. (2013) Prevalence and severity of insomnia in chronic low back pain patients. Rheumatology International, 33(5), 1277-81.

Basics, B. (2011) Understanding Sleep: National Institute of Neurological Disorders and Stroke. 
Bentsen, S. B., Hanestad, B. R., Rustøen, T., Wahl, A. K. (2008) Quality of life in chronic low back pain patients treated with instrumented fusion. Journal of Clinical Nursing, 17(15), 20619.

Boonstra, A. M., Preuper, H. R. S., Balk, G. A., Stewart, R. E. (2014) Cut-off points for mild, moderate, and severe pain on the visual analogue scale for pain in patients with chronic musculoskeletal pain. Pain, 155(12), 2545-50

Buysse, D. J., Ancoli-Israel, S., Edinger, J. D., Lichstein, K. L., Morin, C. M. (2006) Recommendations for a standard research assessment of insomnia. Sleep: Journal of Sleep and Sleep Disorders Research, 29(9), 1155-73.

Crichton, N. Visual analogue scale (VAS). (2001) Journal of Clinical Nursing, 10(5), 706-6.

Freburger, J. K., Holmes, G. M., Agans, R. P., Jackman, A. M., Darter, J. D., Wallace, A. S., et al. (2009) The rising prevalence of chronic low back pain. Archives of Internal Medicine. 169(3), 251-258.

Green, B. N., Johnson, C. D., Snodgrass, J., Smith, M., Dunn, A. (2016) Association between smoking and back pain in a crosssection of adult americans. Cureus, 8(9), 806.

Hurley, D. A., Eadie, J., O'Donoghue, G., Kelly, C., Lonsdale, C., Guerin, S., et al. (2010) Physiotherapy for sleep disturbance in chronic low back pain: a feasibility randomised controlled trial. BMC Musculoskeletal Disorders, 11(1), 70.

Kornu, R., Dao, K.H., Orozco, C., Patel, R.K. (2011) SIGNS AND SYMPTOMS. Medical Secrets, 247.

Lamberg, L. (2007) Several sleep disorders reflect gender differences. Psychiatric News, 42(10), 40.

Marty, M., Rozenberg, S., Duplan, B., Thomas, P., Duquesnoy, B., Allaert, F., et al. (2008) Quality of sleep in patients with chronic low back pain: a case-control study. European Spine Journal, 17(6), 839-44.
Masumian, S., Shairi, M., Hashemi, M. (2013) The Effect of Mindfulness-Based Stress Reduction on Quality of Life of the Patients with Chronic Low Back Pain (persian). Journal of Anesthesiology and Pain, 4(1), 25

Morgenthaler, T., Kramer, M., Alessi, C., Friedman, L., Boehlecke, B., Brown, T., et al. (2006) Practice parameters for the psychological and behavioral treatment of insomnia: an update. An American Academy of Sleep Medicine Report, 29(11), 1415.

Nasab, A.F., Azimi, H. (2008) Study of patterns and subjective quality of sleep and their correlation with personality traits among medical students of Hamadan University of Medical Sciences. Scientific Journal of Hamadan University of Medical Sciences, 15 (1), 11-15

O'Donoghue, G. M., Fox, N., Heneghan, C., Hurley, D.A. (2009) Objective and subjective assessment of sleep in chronic low back pain patients compared with healthy age and gender matched controls: a pilot study. BMC Musculoskeletal Disorders, 10(1),122.

Orhurhu, V. J., Pittelkow, T. P., Hooten, W. M. (2015) Prevalence of smoking in adults with chronic pain. Tobacco Induced Diseases, 13(1), 17-22.

Schrimpf, M., Liegl, G., Boeckle, M., Leitner, A., Geisler, P., Pieh, C. (2015) The effect of sleep deprivation on pain perception in healthy subjects: a meta-analysis. Sleep Medicine, 16(11), 13131320.

Smith, M. T., Haythornthwaite, J. A. (2004) How do sleep disturbance and chronic pain inter- relate? Insights from the longitudinal and cognitive-behavioral clinical trials literature. Sleep Medicine Reviews, 8(2), 119-32.

van de Water, A.T., Eadie, J., Hurley, D.A. (2011) Investigation of sleep disturbance in chronic low back pain: an age-and gender-matched case-control study over a 7-night period. Manual Therapy, 16(6), 550-6. 